A. Pramesh Rao, G. Swarup and Gopal-Krishna, eds.

\title{
The Molonglo Galactic Plane Survey: MGPS2
}

\author{
A. J. Green \\ Astrophysics Department, School of Physics, University of Sydney, \\ NSW 2006, Australia
}

\begin{abstract}
A survey of the southern Galactic Plane is being made in the radio continuum at $843 \mathrm{MHz}$ with the Molonglo Observatory Synthesis Telescope. The area to be covered is $240^{\circ} \leq l \leq 365^{\circ},|b| \leq 10^{\circ}$ with a resolution of $43^{\prime \prime} \times 43^{\prime \prime} \operatorname{cosec}|\delta|$ and a $3 \sigma \mathrm{rms}$ noise of $\leq 5 \mathrm{mJy} / \mathrm{beam}$. This survey (MGPS2) will be used to search for possible new supernova remnant candidates, to image other discrete sources such as HII regions and radio galaxies located behind the Galaxy, and to study the structure of the interstellar medium. Comparison with a first epoch survey (MGPS1) will also be made. Some of the first results from MGPS2 are presented.
\end{abstract}

\section{Introduction}

The Molonglo Observatory Synthesis Telescope (MOST) is an earth-rotation interferometer operating in the radio continuum at $843 \mathrm{MHz}$, with subarcmin resolution (Mills 1981; Robertson 1991). It is a highly redundant synthesis array with an effective collecting area of $18,000 \mathrm{~m}^{2}$. The MOST is an exceptionally sensitive instrument for imaging weak, extended emission with high resolution over a large field of view. Hence, it is ideal for making imaging surveys of the Galactic Plane in order to study both large-scale structure and discrete sources.

\section{The Molonglo Galactic Plane Survey}

There are two Molonglo Galactic Plane surveys: MGPS1 and MGPS2. The first epoch survey (MGPS1) is complete, with images published (Green et al. 1999). The second epoch survey (MGPS2) will cover seven times the area (2500 square degrees) and is presently more than one third complete. Table 1 gives details of the parameters for MGPS2 and the technical characteristics of the MOST.

The data will be mosaiced into $4^{\circ} \times 4^{\circ}$ images, using the same grid for field centres as SUMSS (Bock, Large, \& Sadler 1999). Figure 1 shows an example of the information expected from each mosaic. A montage in Galactic co-ordinates will also be constructed. Images from MGPS1 can be found on the website http://www.astrop.physics.usyd.edu.au/MGPS. MGPS2 images will be released as soon as possible, after appropriate quality checks and calibration. 


\begin{tabular}{ll}
\hline Galactic longitude range & $240^{\circ} \leq l \leq 365^{\circ}$ \\
Galactic latitude range & $|b| \leq 10.0^{\circ}$ \\
Field size & $160^{\prime} \times 160^{\prime} \operatorname{cosec}|\delta|$ \\
Number of fields & 621 \\
Completed November, 1999 & $38 \%$ \\
& \\
Centre frequency of observations & $843 \mathrm{MHz}(\lambda=36 \mathrm{~cm})$ \\
Bandwidth & $3 \mathrm{MHz}$ \\
Synthesized beam & $43^{\prime \prime} \times 43^{\prime \prime} \operatorname{cosec}|\delta|$ \\
Dynamic range (maximum) & $\sim 250: 1$ \\
RMS noise $(1 \sigma)$ after $12 \mathrm{hr}$ & $1-2 \mathrm{mJy} / \mathrm{beam}$ \\
Surface brightness sensitivity & $\sim 1 \mathrm{~K}(1 \sigma)$ \\
\hline
\end{tabular}

Table 1. Characteristics of MGPS2 and the MOST

\section{Scientific Goals}

The images show many thousands of discrete sources, both unresolved and extended, together with a complex web of filamentary structures. The principal science outcomes expected are:

- Inventory of Supernova Remnants (SNRs): SNRs are a major source of energy and material for the interstellar medium (ISM). In turn, the environment of each SNR largely determines its evolution. Although shortlived, these end products of star formation provide a valuable snapshot of ISM conditions. The MOST has been a very effective instrument for identifying new SNRs.

- Imaging HII Regions: The faint, highly-structured extended envelopes around many HII regions will be mapped and the results correlated with mid-infrared data, which trace the distribution of polycyclic aromatic hydrocarbons found round many ionised hydrogen shells (Cohen \& Green 1999 , in preparation).

- Unraveling the nature of the Filaments: Large-scale filamentary structure is seen over much of the Galactic Plane. This may be related to superbubbles or merely fluctuations in the ISM. Many are clearly generated by thermal processes, but this may not be the whole story. Comparison with HI data (Dickey, 1999) may clarify the emission mechanisms involved.

- Search for Galaxies in the Zone of Avoidance: As well as background galaxies and normal spirals (both typically unresolved by MOST), there are also many extended sources with the morphology of radio galaxies. Obscuration makes identification using standard optical techniques difficult (Juraszek, Green \& Hunstead 1999, in preparation).

- Mapping the Population of Unresolved Sources: About 15\% of the unresolved sources are probably Galactic. Searches for young SNRs and plan- 


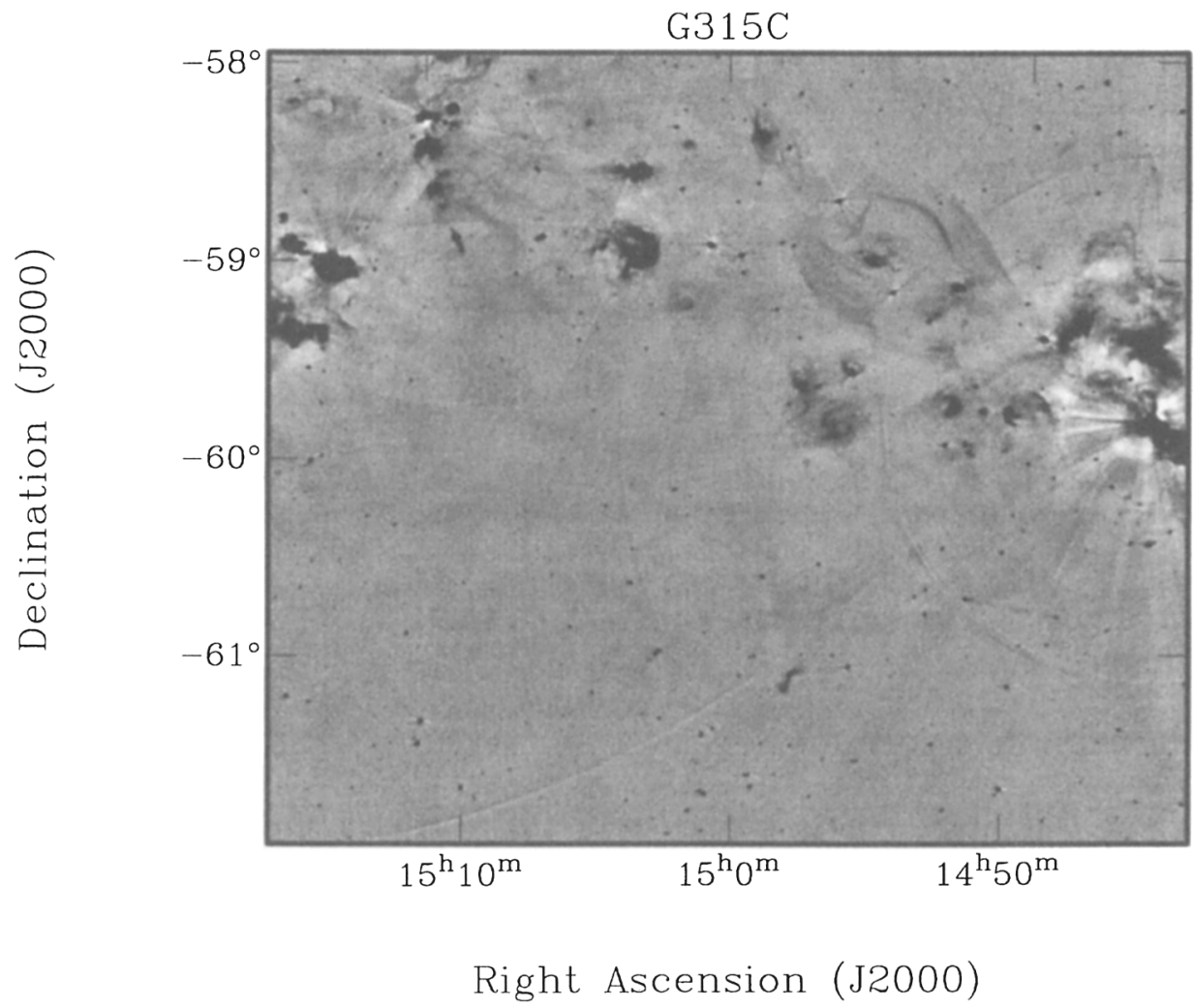

Figure 1. A mosaic of part of the Galactic Plane near longitude $l=318^{\circ}$. The weakest emission reliably detected is $3 \mathrm{mJy} / \mathrm{beam}$ and the strongest source in the image is the HII region on the western edge, which has a peak of $4.1 \mathrm{Jy}$.

etary nebulae are in progress. Comparison with MGPS1 may reveal some long-term variable sources.

Acknowledgments. The MOST is operated by the School of Physics, with the support of the Australian Research Council and the Science Foundation for Physics within the University of Sydney.

\section{References}

Bock, D. C.-J., Large, M. I., \& Sadler, E. M. 1999, AJ, 117, 1578

Dickey, J. M., McClure-Griffiths, N., Gaensler, B. M., Green, A. J., Haynes, R. F., \& Wieringa, M. H. 1999, IRTS, Tucson, AZ

Green, A. J., Cram, L. E., Large, M. I., \& Ye, T. 1999, ApJS, 122, 207

Mills, B. Y. 1981, Publ. Astr. Soc. Australia, 4, 156

Robertson, J. G. 1991, Australian J. Phys., 44, 729 